\title{
Cervical spondylotic myelopathy: an update
}

\section{Editorial}

\author{
Ronald H. M. A. Bartels • Michael G. Fehlings • \\ Kuniyoshi Abumi
}

Received: 2 November 2013/Revised: 3 November 2013/ Accepted: 6 November 2013/Published online: 22 November 2013

(C) Springer-Verlag Berlin Heidelberg 2013

It was an honor to be invited by the Editor of the European Spine Journal to create a supplement about cervical spondylotic myelopathy (CSM), in recognition of our close relationship with the European, North American and Asia Pacific sections of the Cervical Spine Research Society (CSRS). In particular, the European section of the CSRS is one of the societies that have supported this Journal issue from the beginning.

CSM is the commonest cause of spinal cord impairment in adults worldwide. With an aging population, improved diagnostic and surgical techniques and emerging evidence for changes in treatment protocols, CSM is indeed a very interesting and timely issue. As summarized in this special focus issue, a diagnosis of CSM should be made early in the course of the disease, the normal evolution without treatment and after surgical intervention should be predicted, a surgical treatment should be tailored towards the problems of the individual patient, and an adequate postoperative rehabilitation program should be provided.

While composing this supplement it became clear for us that there is a need for a concise up-to-date summary of the evidence to guide treatment for CSM. Although the clinical entity is known for a long time, its exact incidence or

R. H. M. A. Bartels ( $\square)$

Department of Neurosurgery, Radboud University Medical

Center, Geert Grooteplein-Zuid 10, 6525 GA Nijmegen,

The Netherlands

e-mail: Ronald.bartels@radboudumc.nl

M. G. Fehlings

Toronto Western Research Institute, University of Toronto, University Health Network, Toronto, Canada

K. Abumi

Sapporo Orthopaedic Hospital, Center for Spinal Disorders,

Sapporo, Japan prevalence is still not known. Imaging techniques are evolving, as are current concepts in pathophysiology. Laminectomy was and still remains a common option for dorsal decompression. However, other techniques including laminoplasty, which seeks to retain some degree of segmental motion and laminectomy and instrumented fusion, which enables handling of instability and prevention of post-decompression kyphosis, are important options, which require careful consideration.

With this supplement we seek to provide a concise and factual overview of several aspects related to CSM. We can conclude that sound state of the art evidence is emerging. Therefore, this supplement is a compelling invitation to investigators and reviewers for submitting, respectively accepting only results of studies that were performed according to modern standards.

Finally, we the Guest Editors are very much indebted for the important contributions of the colleagues who have written the articles in the focus issue. We hope you enjoy reading this supplement and also will use it as a reference for your future scientific activities.

Conflict of interest R. Bartels and K. Abumi do not have any potential conflict of interest. M. Fehlings received royalties from Depuy for the Discover Disc technology. 